\title{
OPEN NMR microsystem for label-free characterization of 3D nanoliter microtissues
}

\author{
Marco Grisi ${ }^{1 凶}$, Gaurasundar M. Conley ${ }^{1}$, Kyle J. Rodriguez ${ }^{2}$, Erika Riva ${ }^{3}$, Lukas Egli ${ }^{4}$, \\ Wolfgang Moritz ${ }^{4}$, Jan Lichtenberg ${ }^{4}$, Jürgen Brugger ${ }^{2} \&$ Giovanni Boero $^{2}$
}

Performing chemical analysis at the nanoliter $(n L)$ scale is of paramount importance for medicine, drug development, toxicology, and research. Despite the numerous methodologies available, a tool for obtaining chemical information non-invasively is still missing at this scale. Observer effects, sample destruction and complex preparatory procedures remain a necessary compromise. Among non-invasive spectroscopic techniques, one able to provide holistic and highly resolved chemical information in-vivo is nuclear magnetic resonance (NMR). For its renowned informative power and ability to foster discoveries and life-saving applications, efficient NMR at microscopic scales is highly sought after, but so far technical limitations could not match the stringent necessities of microbiology, such as biocompatible handling, ease of use, and high throughput. Here we introduce a novel microsystem, which combines CMOS technology with 3D microfabrication, enabling $\mathrm{nL}$ NMR as a platform tool for non-invasive spectroscopy of organoids, 3D cell cultures, and early stage embryos. In this study we show its application to microlivers models simulating non-alcoholic fatty liver disease, demonstrating detection of lipid metabolism dynamics in a time frame of 14 days based on 117 measurements of single 3D human liver microtissues.

Nuclear magnetic resonance (NMR) is a powerful spectroscopic tool for non-invasive investigations of the chemistry in intact living matter ${ }^{1,2}$. In the last decades, both Magnetic Resonance Imaging (MRI) and NMR spectroscopy have been key enablers for several lifesaving diagnostic applications ${ }^{3-8}$ as well as groundbreaking fundamental research on animal and human bodies ${ }^{9,10}$. However, due to limitations of the current commercial hardware, magnetic resonance cannot be routinely applied to in-vivo and in-vitro studies at the nanoliter (nL) scale, typical of microorganisms and cell cultures. Indeed, micro-NMR is a longstanding technical challenge, and numerous researchers have proposed methods to achieve it, mainly using micro solenoids ${ }^{11,12}$, planar micro coils $^{13}$, magic angle coil spinning (MACS) probes at ultra-high fields ${ }^{14}$, reaching sample volume sizes of about 10 $\mathrm{nL}$. More recently, NMR spectroscopy of eggs of microorganisms having a volume of just $0.1 \mathrm{~nL}$ was reported in a relatively weak field strength of $7 \mathrm{~T}$. Such advancement was possible thanks to a novel approach based on complementary metal-oxide semiconductor (CMOS) microchips that delivered high sensitivity, robustness, and a local and easily accessible sensing region ${ }^{15}$. All these works pushed NMR to the nL range, but sample handling issues and a high level of complexity remained an obstacle to high throughput studies with sufficient statistics, essential for biology, preventing widespread adoption. In this work we present an innovative device that combines the advantages of CMOS-based NMR probes with an unprecedented ease of use, allowing investigations of $\mathrm{nL}$ biological entities with a satisfactory statistic for the first time.

To show these capabilities, we here describe a first study using nL NMR on 3D microtissues (MTs), microscopic cell aggregates that mimic features and functionalities of their macroscopic counterparts. MTs have been introduced as a mean to simulate and probe disease conditions by allowing high throughput drug screening without need for biopsies of sick individuals, to better understand the mechanisms by which diseases operate and progress, and to reduce the need for animal testing, all of which could contribute to an accelerated development of new therapeutic treatments ${ }^{16-19}$. In this study we focus on human liver MTs (hLiMTs), specifically treated to simulate non-alcoholic fatty liver disease (NAFLD) $)^{17,18}$. NAFLD has become a global medical issue for $10-40 \%$ of adults from different demographics worldwide, and the mechanisms by which this disease progresses still eludes researchers ${ }^{20,21}$. This progressive disease is characterized by the initial accumulation of lipids in hepatocytes, predominantly in the form of triglycerides. The homeostasis of these hepatic lipids depends greatly on several

${ }^{1}$ Annaida Technologies SA, Lausanne, Switzerland. ${ }^{2}$ Microsystems Laboratory, École Polytechnique Fédérale de Lausanne (EPFL), Lausanne, Switzerland. ' ${ }^{3}$ ervice de Gastro-Entérologie et D'hépatologie, CHUV, Epalinges, Switzerland. ${ }^{4}$ InSphero AG, Schlieren, Switzerland. ${ }^{\square}$ email: marco.grisi@annaida.ch 

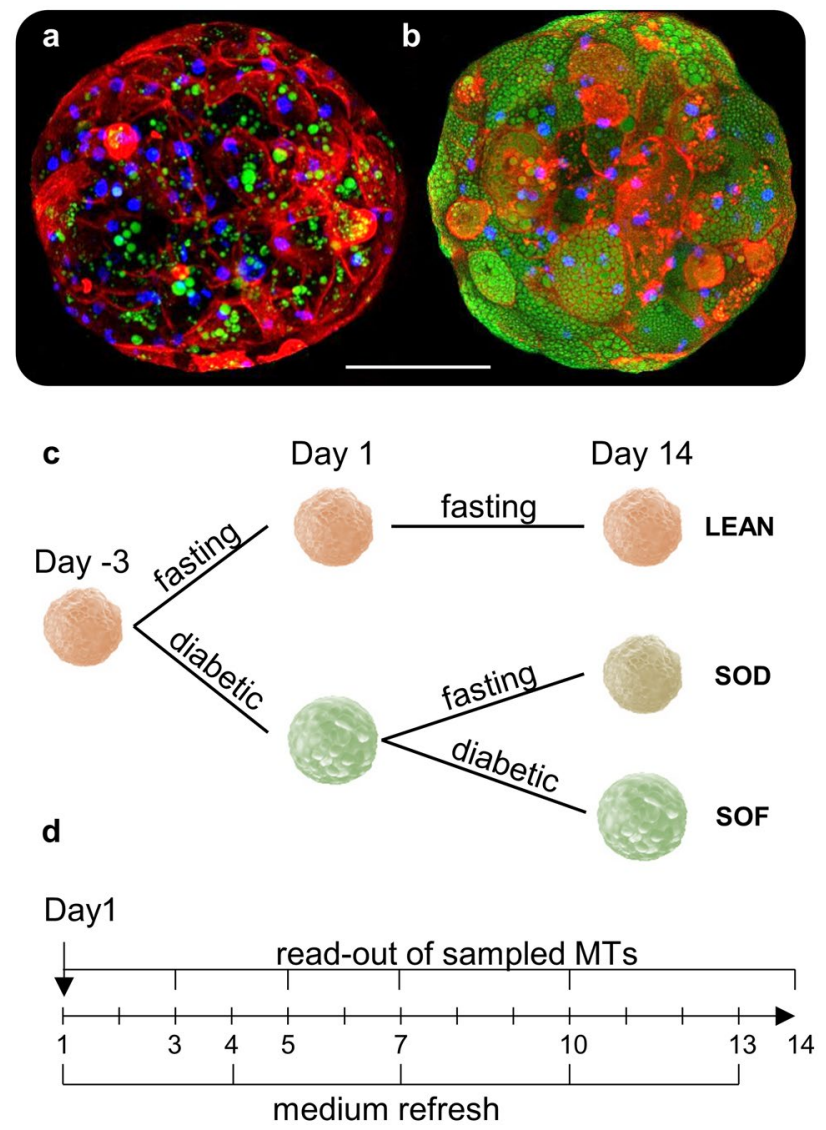

Figure 1. Samples and experimental design. Representative confocal images of human liver microtissues generated in-vitro after incubation in medium containing bovine serum albumin (BSA) (a) or oleic acid coupled to BSA (OA/BSA) (b) for 7 days. MTs were fixed and stained with Hoechst (nuclei, blue), CellMask (cell membrane, red) and Nile Red (lipids, green). The images clearly distinguish between a lean and fatty liver phenotype reminiscent of microvesicular steatosis. Scalebar $=100 \mu \mathrm{m}$. (c) Scheme depicting culture conditions of liver microtissues, starting after spheroid formation (day -3) and incubation until day 14 days with different media compositions, i.e. fasting medium (low insulin, low glucose) and diabetic medium (high insulin, high glucose) with fractionated plasma LDL. The three resulting experimental groups are lean livers (LEAN), steatotic livers "on diet" (SOD), steatotic livers "on fat" (SOF). (d) Read-out and medium refreshing time points.

pathways such as fatty acid uptake, de novo synthesis, oxidation, and very low-density lipoprotein (VLDL) secretion $^{22,23}$. The regulation of these pathways and their metabolic profiles are fundamental to the progression of NAFLD, therefore, many researchers have focused their efforts in proteomic and lipidomic studies ${ }^{24,25}$. While these studies have been instrumental in understanding NAFLD, the techniques used rely on invasive methods, such as biopsies, and destructive analyses. The situation is analogous for MTs, for which an informative, quantitative and non-invasive technique, capable of characterizing individual specimens, is still missing, and NMR metabolomics studies have suggested MR is a suitable technology to probe NAFLD ${ }^{5,16,26-28}$. In this study we successfully utilize NMR spectroscopy on single liver MTs and demonstrate the observation of a dynamic fatty acid metabolism associated to a diet switch inducing recovery from a steatotic condition.

\section{Results and discussion}

In our experiments we use hLiMTs generated in-vitro from human hepatic cells of healthy donors (3D Insight Human Liver Microtissues, InSphero AG, Switzerland $)^{17,18}$. MTs are then exposed to different dietary regimes: a fasting diet is used to culture the livers in a lean state (as control), while a diabetic (fatty) diet induces steatosis (see "Methods"). The images (Fig. 1a,b) show a comparison between a lean and a steatotic micro-liver, with lipids stained in green. Clearly, the steatotic MT is characterized by an accumulation of lipids, main and typical manifestation of NAFLD ${ }^{20,21}$. In our study a dynamic metabolism is induced by a diet switch: we start at Day1 with a steatotic and a lean experimental group, and we create a third group by administering a fasting diet to steatotic livers for 14 days (Fig. 1c). The three resulting experimental groups are LEAN (healthy livers in fasting medium), SOD (steatotic livers “on diet", i.e. in fasting medium), SOF (steatotic livers "on fat", i.e. in diabetic medium). During the course of the experiments, media are routinely refreshed to maintain MT viability, and small batches of MTs are collected and analyzed in 6 time points as indicated in Fig. 1d (see "Methods" for experimental protocols). 
a

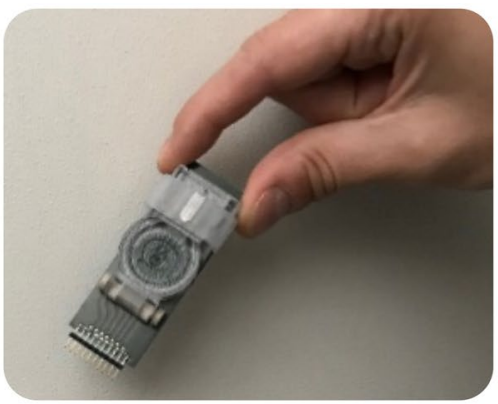

C
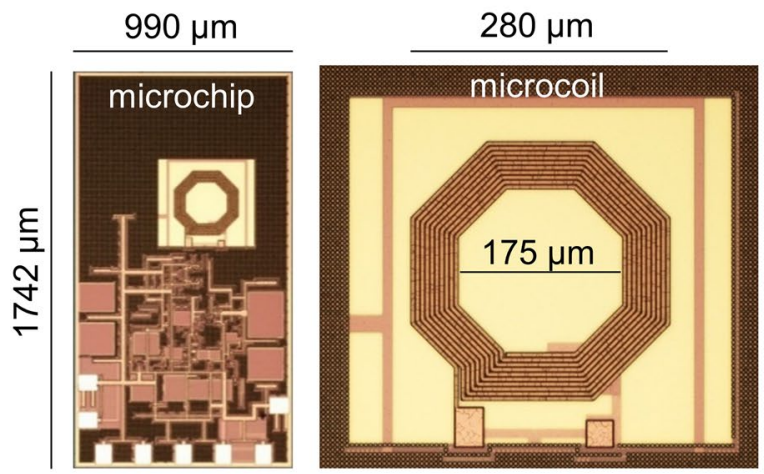

e

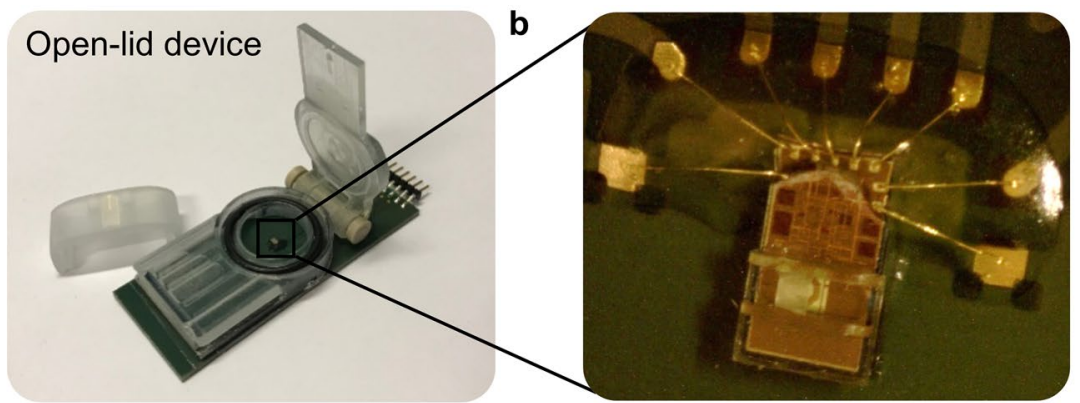

d

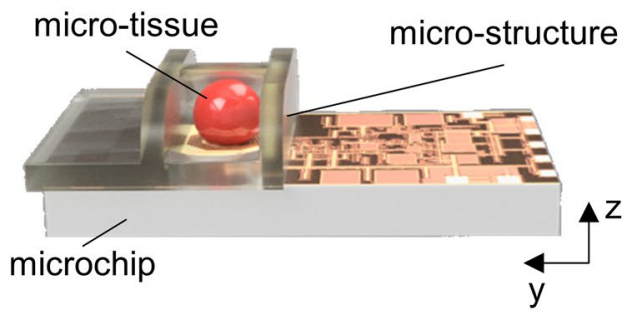

micro-tissue
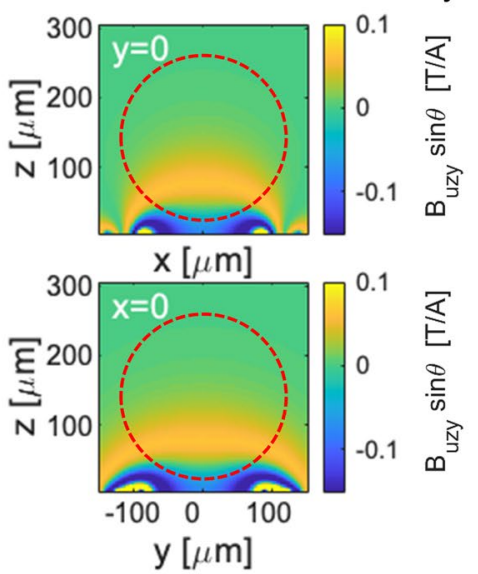

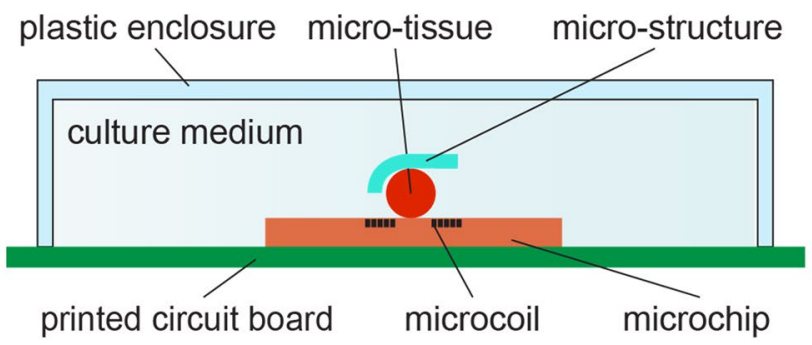

Figure 2. CMOS-based NMR probes for nL 3D MTs. (a) Photographs of our CMOS-based NMR sensor $(6 \times 2.5 \mathrm{~cm})$ designed for in-vivo/in-vitro experiments at the $\mathrm{nL}$ scale. Left: sensor closed and ready for being inserted in the magnetic field. Right: sensor with open lid. (b) Photograph of the micro-system used to perform NMR spectroscopy of MTs. (c) Photographs of the CMOS microchip and its microcoil. The coil region appears with a different color due to the absence of metal density filling in order to minimize parasitic effects. (d) 3D rendering at scale of the micro-system and sample. The sample is approximated by a sphere having a diameter of $240 \mu \mathrm{m}$. In the indicated reference frame, the static field $\mathrm{B}_{0}$ and gravity are along the $\overline{\mathrm{x}}$ axis. (e) Cross-section schematics of the micro-system, defined as crossing transversally the chip at the microcoil center. The curved shape of the sample-positioning micro-structure enables placement of MTs of different sizes in the most sensitive area of the microcoil. (f) Maps of sensitivity of the integrated microcoil in experimental conditions $(\tau=20 \mu \mathrm{s}, i=11 \mathrm{~mA})$ at $\mathrm{y}=0$ and $\mathrm{x}=0$ cross-sections. The static $B_{0}$ field is oriented along the $\overline{\mathrm{x}}$ axis, while the $\overline{\mathrm{Z}}$ axis is perpendicular to the coil surface. The octagonal loops of the coil are approximated with circular ones. The unitary field $B_{\mathrm{u}}$, defined as the field produced by a current of $1 \mathrm{~A}$ in the coil loops, is computed via BiotSavart. The component orthogonal to $B_{0}$ (i.e. $B_{\text {uzy }}$ ) is then considered to compute the nutation angle $\theta$. Red dashed circular shapes indicate the sample as in (d-e). The excitation current $i=11 \mathrm{~mA}$ is deduced by matching maximum signal intensity from experiments and sensitivity maps computation. This is in line with electronic simulations of the CMOS circuit in Cadence.

Figure 2 shows photographs and schematics of the CMOS-based NMR sensor (Annaida Technologies SA, Switzerland), consisting of a microsystem enclosed in a 3D printed plastic container (Veroclear, Connex500, Computer Aided Technology) holding the culture medium. The container has an openable lid, which presses on an o-ring (black in Fig. 2a) to seal the chamber and limit medium evaporation during measurements when closed, and allowing for quick sample access when open. The sensor connector is plugged into a dedicated receptacle, containing an electronic interface to the external console, prior insertion in the magnetic field (see S1). In this configuration, the sensor can be used as a plug and play device for an eased loading procedure.

The microsystem (Fig. 2b) is made by combining a CMOS chip (Fig. 2c) with a 3D micro-structure made with a two-photon polymerization 3D printing technique (Photonic Professional GT by Nanoscribe GmbH, $\mathrm{DE}$ ). This method, having a resolution below $1 \mu \mathrm{m}^{3}$, allows fabricating structures that can easily be adapted to specific sample sizes and shapes. In this work the structure was specifically designed to position and hold MTs 
that are well approximated by spherical shapes (Fig. 1a, b). Our group had previously used this same fabrication technique to realize closed micro-channels for in-vivo measurements ${ }^{29}$. However, this previous system required a laborious mounting procedure, preventing the systematic accumulation of large, statistically relevant data sets. In the current device, a user-friendly design enables rapid sample placement for higher throughput experiments. The CMOS chip is wire bonded to the printed circuit board and the wires are covered with a protective glue. The sample-positioning micro-structure is then permanently attached to the chip surface to create a hosting spot for the MTs aligned on top of the integrated microcoil (Fig. 2d, e). Such pre-mounted micro-system is robust and allows for quick sample loading $(<5 \mathrm{~min})$ with the sole use of a micro-pipette and a stereomicroscope. The whole set of measurements presented in this work was obtained by using only two identical sensors (see "Methods").

Figure $2 \mathrm{f}$ shows the sensitivity maps of the device as computed in the experimental conditions with a finite element model. Owing to the relatively long pulse used in the experiments $(\tau=20 \mu \mathrm{s}, \mathrm{i}=11 \mathrm{~mA})$ a negative phase is originated close to the chip surface, which results in a reduction of the contribution to the total signal from the portion of medium close to the coil loops and outside the sample region by cancellation effects. In this configuration, the central part of the coil (i.e., where the sample is placed) has mainly a positive phase contribution, which constructively adds up in the total signal. From complementary experiments, we observed that the volume of the sample varies over time (see S2). Since in our system only a portion of the sample is analyzed (orange in Fig. 2f), the relation between signal intensity and volume is not expected to be linear. However, with the use of sensitivity maps, it is possible to estimate how sample volume affects the NMR signal and correct the data by factoring it out (see "Methods"). This is needed only if absolute quantities are compared.

Figure 3 shows spectroscopy results of single MTs obtained with our CMOS-based sensor in a field strength of $7 \mathrm{~T}$. Typical spectra are shown for acquisition times of $1 \mathrm{~h}$ (Fig. 3a) and $10 \mathrm{~min}$ (Fig. 3b). The spectral resolution is about $0.2 \mathrm{ppm}$, slightly better than what observed by in-vivo liver studies ${ }^{30}$, and pronounced peaks are visible in the region between 0 and $6 \mathrm{ppm}$. In order to acquire a sufficiently large set of data to infer statistically significant conclusions, we fixed an experimental time of $10 \mathrm{~min}$ per single MT and performed several measurements of single MTs in the time points indicated in Fig. 1d. Figure 3c shows the 21 measurements acquired at Day14 for the three experimental groups, while the complete collection of the 117 spectra of single MTs is reported in S3. From averaged spectra at Day1 (Fig. 3d) and Day14 (Fig. 3e) it is evident that the SOD evolves to differentiate from SOF, expressing a phenotype more similar to LEAN in the long term. In the following, we interpret some of the peaks in our spectra to define numerical quantities (biomarkers) that can be used to follow this dynamic.

Previous literature reports have combined the use of ${ }^{1} \mathrm{H}$ NMR spectroscopy on intact liver tissues and on extracts to determine the speciation of metabolites ${ }^{31}$ and to assess the role of lipids in liver quality ${ }^{32}$. Further evidence from in-vivo MRS of rat liver ${ }^{30}$ and NMR spectroscopy of oils ${ }^{33}$ suggests that the prominent signals shown in Fig. 3 correspond predominantly to lipids. With the scope of identifying efficient biomarkers, we restrict the analysis only to those signals that resonate sufficiently far from water to avoid overlap with its baseline. As discussed in S4, S5, background and MT measurements indicate that the region ranging from 0 to $3.2 \mathrm{ppm}$ can be considered for our purpose. Since metabolites are expected, in this spectral region, to give signals with a signal-to-noise ratio (SNR) lower by about one order of magnitude with respect to lipids ${ }^{31}$, we can consider their contribution negligible. To further corroborate this observation, lipidomic data obtained via mass spectroscopy of MTs extracts confirm that lipids are present in concentrations ranging from 0.1 to $1 \mathrm{M}$ (see S6), in agreement with the observed $0.5 \mathrm{M}$ of $-\left(\mathrm{CH}_{2}\right)_{\mathrm{n}}$ deduced from the signal amplitude at $1.3 \mathrm{ppm}$. These lipids are predominantly in the forms of triglycerides (TAGs), cholesteryl ester derivatives (CEs), and free fatty acids (FAs). While it is difficult to determine exact speciation with our SNR and spectral resolution, it is still possible to ascertain the types of functional groups and deduce information related to the degree of unsaturation of the lipids in the hLiMTs $^{30,33}$ (see Table S1). As previously reported, a reliable way to obtain numerical values for statistical evaluations is to integrate over defined spectral regions ${ }^{32}$. In our case we identify three spectral regions of interest ranging from 1.18-1.46 ppm (integral $\mathrm{L}_{1}$ ), 1.80-2.20 ppm (integral $\mathrm{L}_{2}$ ), and 2.50-3.00 ppm (integral $\mathrm{L}_{3}$ ), which respectively represent aliphatic chains corresponding to the presence of lipid signatures from fully saturated $\left(\mathrm{L}_{1}\right)$, mono- $\left(\mathrm{L}_{2}\right)$ and poly- $\left(\mathrm{L}_{3}\right)$ unsaturated fatty acids (see $\mathrm{S} 7, \mathrm{~S} 8$ ).

Figure 4 shows experimental results and statistical significance of four different biomarkers defined to interpret the biological state of NAFL hLiMTs. Three of these markers are defined as ratios of the integrals $\mathrm{L}_{\mathrm{i}}$ and represent relative concentrations of the aforementioned molecular groups. It is worth to note that these quantities, since defined by ratios of integrals computed from spectra of the same MT, are independent from the sample volume. In order to measure the biomarkers variability due to instrumental error (originating from electronic noise) and systematic errors (originating from sample placement, probe positioning, etc....), 12 additional measurements were performed on two MTs (6 each). The result of this investigation, presented in S9, shows that the variability due to error sources is well below the one observed across the different experimental groups, indicating that the defined markers provide a reproducible and sufficiently precise measurement to represent each MT. Figure $4 a-c$ show a direct comparison between values at the first and last time points. The SOD, significantly different from LEAN at Dayl, approaches it differentiating from SOF in the long term. Figure 4e shows the time evolution of $\mathrm{L}_{3} / \mathrm{L}_{1}$ monitored at all time points, where we can see its dynamics evolving gradually, but with a stepwise behavior between Day7 and Day10. Such behavior is also true for $\mathrm{L}_{2} / \mathrm{L}_{1}$, while for $\mathrm{L}_{3} /$ $\mathrm{L}_{2}$ a change is already visible between Day5 and Day7 (see S10). A fourth marker, in analogy with conventional MRI-PDFF (Proton Density Fat Fraction, today's gold standard and Endpoint in NASH trials) ${ }^{34}$ compares the content of $-\left(\mathrm{CH}_{2}\right)_{\mathrm{n}}$ groups to those found in LEAN at Dayl. This marker, named Proportional Lipid Content (i.e. PLC), is a quantitative measurement of the saturated protons and it is corrected for MT volume variations. Figure 4d shows that also PLC can well represent a dynamic evolution of the SOD from a steatotic to a lean status. Figure $4 \mathrm{f}$ shows the overall dynamics, where we can see a gradual approach over time of the SOD from a lipid accumulation phase to a lean condition. The SOF, on the other hand, shows saturation of lipid content, reaching a maximum value already by Day 5 and remaining constant after that. In fact, the SOF showed no improvement 

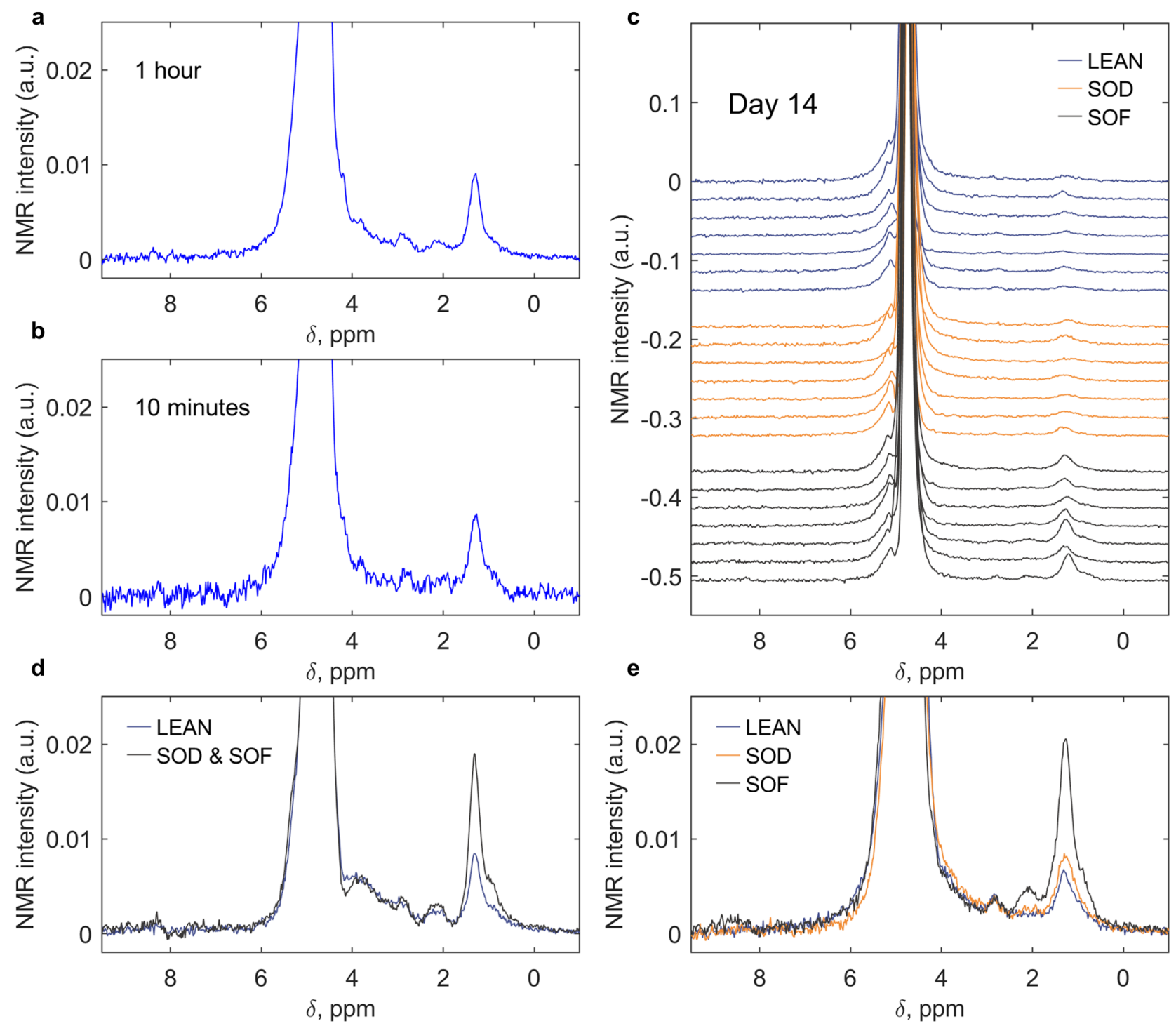

Figure 3. NMR spectroscopy of 3D microtissue cultures. (a) $1 \mathrm{D}^{1} \mathrm{H}$ NMR spectrum obtained from a steaotic MT exposed to fasting medium (SOD) at Day10 over a measurement time of $1 \mathrm{~h}$, i.e. averaging 1800 scans. (b) $1 \mathrm{D}^{1} \mathrm{H}$ NMR spectrum obtained from the same SOD MT as in (a) over a measurement time of $10 \mathrm{~min}$, i.e. averaging 300 scans. (c) The complete series of measurements of individual MTs at Day14, where 7 measurements were collected for SOD, LEAN, and SOF MTs. Each measurement is obtained in 10 min time (i.e., averaging 300 scans). (d, e) NMR spectra averaged by experimental group at Dayl (d) and Day14 (e).

from its steatotic state, while SOD MTs exhibited recovery reaching a state similar to that of LEAN. The data also reveal that while the PLC signal of SOD decreases between Day 7 and Day 14 towards lean values, L3/L1 shows a significant increase during the same period. This demonstrates that MTs under a fasting regimen not only reduce fatty acid content, but also gain in polyunsaturated fatty acid (PUFA) to saturated fatty acid (SFA) ratio. Similar alterations in hepatic PUFA/SFA ratio have been described in NAFLD/NASH patients compared to healthy control ${ }^{25}$. In summary, these data suggest that MTs are capable of reproducing a behavior similar to that exhibited by livers in-vivo, where studies relying on biopsies have shown that NAFL patients accumulated $5-10 \times$ the amount of fatty acids with an altered fats composition ${ }^{24}$, and fasting diets reduced lipid accumulation ${ }^{35}$. Similarly, in this study, the LEAN exhibited far less lipid content than SOF livers, and SOD MTs recovered to a lean status when exposed to a fasting medium.

\section{Conclusion}

In this work we have demonstrated and validated a device concept that enables NMR on single biological entities at the nanoliter scale. Its simplicity of use, sensing performance, robustness and versatility makes this technology suitable for extended high throughput non-invasive investigations of intact microscopic biological samples. The use of this CMOS-based NMR device is demonstrated in an exemplary study, detecting fatty acid metabolism dynamics, label-free, from more than 100 measurements of single nL human liver microtissues. The flexibility of 


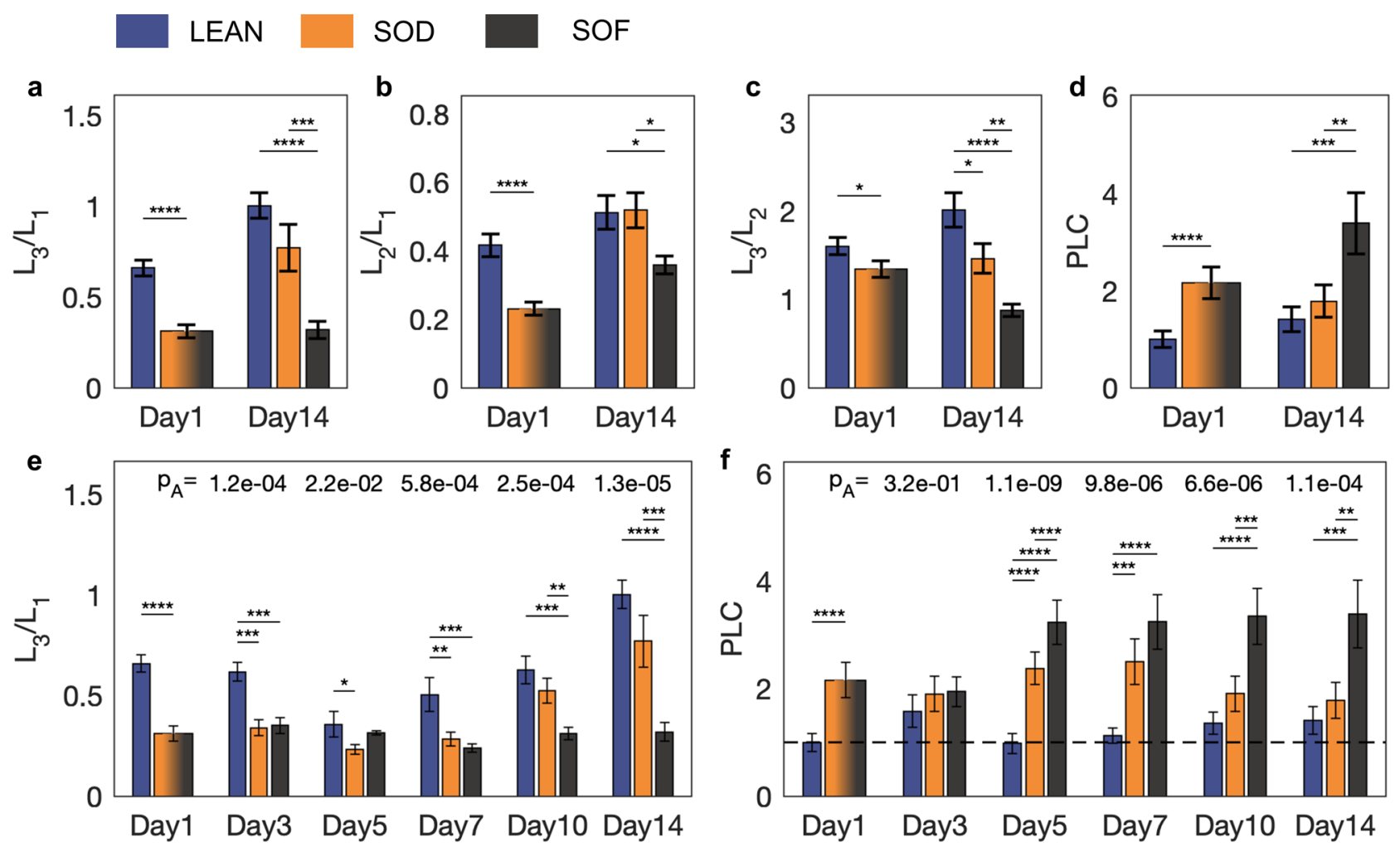

Figure 4. Label-free detection and evolution of lipids. Data are computed from spectra obtained by averaging 300 scans (i.e., 10 min measurement time). At each time point biomarkers of MTs are grouped as LEAN, SOD, SOF. At Day1, SOD and SOF coincide. 7 data points are acquired for SOD, except 5 data points at Day3. 7 data points are acquired for SOF, except 5 data points at Day14. For LEAN, 7 data points are acquired at Day1 and Day14, 6 data points at Day7 and Day10, 5 data points at Day3 and Day5. In total, 117 experiments are performed on single MTs. At Day1, significance is computed with a student t-test. From Day3, one-way ANOVA ( $\mathrm{p}$ value ' $\mathrm{p}_{\mathrm{A}}$ ' is indicated) and Tukey-Kramer tests are applied (biomarkers distributions are shown in S11). ${ }^{\star} P<0.05,{ }^{\star *} P<0.01,{ }^{* *} P<0.001,{ }^{* * *} P<0.0001 . \mathrm{L}_{1}$ : integral area from 1.18 to $1.46 \mathrm{ppm}$. $\mathrm{L}_{2}$ : integral area from 1.8 to $2.2 \mathrm{ppm}$. $\mathrm{L}_{3}$ : integral area from 2.5 to 3 ppm. PLC: $\mathrm{L}_{1} / \mathrm{L}_{1 \text { LEAN_Dayl }}$. PLC values are corrected for volume variation to represent concentration values (see "Methods"). Direct comparison at Dayl and Day14 of: (a) $\mathrm{L}_{3} / \mathrm{L}_{1}$ (b) $\mathrm{L}_{2} / \mathrm{L}_{1}$ (c) $\mathrm{L}_{3} / \mathrm{L}_{2}$ (d) PLC. Complete time evolution of: (e) $\mathrm{L}_{3} / \mathrm{L}_{1}$ (f) PLC.

the fabrication methodology makes CMOS-based micro-NMR a promising platform for more widespread use of the technique in different research environments. Such an advancement would enable non-invasive chemical analysis of biological entities that are currently out of reach, opening to novel applications based on new characterization criteria for a multitude of microorganisms, organoids, microtissues, and even early stage embryos, with great potential impact in medicine, drug development, and fundamental biochemical research.

\section{Methods}

Experiments and protocols were approved by the SV-biosecurity unit committee of the École Polytechnique Fédérale de Lausanne and carried out in accordance with the experimentation guidelines of the institution.

NMR experimental details. NMR experiments were performed in the $54 \mathrm{~mm}$ room temperature bore of a Bruker $7.05 \mathrm{~T}(300 \mathrm{MHz})$ superconducting magnet. The electronic setup is noticeably similar to the one described in detail in Ref. ${ }^{36}$. In this realization, the RF sources, DAQ, and TTL pulses are generated by a modified Tecmag Scout console, whose GUI is used to manage the experimental parameters. Two different frequencies are used in transmit and receive mode with a resulting down-conversion at about $11 \mathrm{kHz}$. Transmit frequency is fixed at the Larmor frequency. All experiments were performed with a repetition time of $2 \mathrm{~s}$, a pulse length of $20 \mu \mathrm{s}$, an acquisition time of $200 \mathrm{~ms}$ with 8192 data points, and at a temperature of $27.5 \pm 0.5^{\circ} \mathrm{C}$ (measured with a calibrated thermocouple at the site of the sample). Prior measurements at each time point the magnetic field is shimmed with a probe without sample (i.e., a sample of culture medium) and left untouched for the day. The achieved spectral resolution with the culture medium is about $0.05 \mathrm{ppm}$ full-widht-at-half-maximum.

NMR data analysis. During the automated data analysis, all spectra are processed with strictly the same algorithm. The time domain data were post-processed by applying an exponential filter with decay time of $100 \mathrm{~ms}$ prior FFT. A frequency window going from 5 to $16 \mathrm{kHz}$ is considered for analysis. An automatic algorithm is used to phase the spectra by maximizing the sum of the real part. The frequency axis is transformed 
to ppm units, and the maximum value of the water peak is aligned at $4.75 \mathrm{ppm}$. After baseline correction, the biomarkers are computed as explained in S8 and standard statistical analysis is applied to the resulting values. Overall, this study is based on 117 spectra of single micro-tissues, plus 12 spectra obtained on two micro-tissues in order to measure experimental systematic and instrumental errors.

On-chip microcoil details. The on-chip microcoil is realized using 4 of the 6 metal layers available in the CMOS technology. 10 loops with pitch of $5 \mu \mathrm{m}$ are used on the top metal (metal 6), and 12 loops with pitch of $10.6 \mu \mathrm{m}$ are equally distributed in three layers (metal 5,4,3). The loops are connected in series. The internal diameter of the coil is $180 \mu \mathrm{m}$.

Correction for volume variation. With the use of sensitivity maps in Fig. $2 \mathrm{f}$, it is possible to estimate how sample volume affects the NMR signal and therefore correct data factoring out sample volume dependency. For instance: by integrating the sensing map over the sample region shown in Fig. $2 \mathrm{f}$ (dashed red shape) we can compute the total signal from a sample having a diameter of $240 \mu \mathrm{m}$. The same calculation over a sample volume having a diameter of $200 \mu \mathrm{m}$ shows that the signal intensity decreases by about $25 \%$. Therefore, if absolute quantities deduced from spectra are compared from these two situations, a correction factor of 0.75 should be taken into account. When absolute quantities are compared (PLC in Fig. 4), this procedure is used to correct for sample volume variations. The numerical correction factors for MTs on fasting and diabetic medium, deduced from S2 and Fig. $2 \mathrm{f}$ at the six time points in Fig. $1 \mathrm{~d}$, are respectively: $\mathrm{C}_{\text {fasting }}=[1,0.98,0.96,0.94,0.82,0.6]$; $\mathrm{C}_{\text {diabetic }}=[1,0.98,0.96,0.94,0.84,0.74]$.

Statistical analysis. Student's t-test, or one-way ANOVA and Tukey-Kramer tests were used to calculate significance levels between groups with a Matlab program. Graphs and figure legends are annotated with the level of significance between the test groups.

In vitro generation of MTs and culture. All spheroid hLiMT used in this study were 3D InSight Human Liver Microtissues (InSphero AG, Schlieren, Switzerland) produced according to the patent-pending protocol (WO2015/158777A1), composed of primary human hepatocytes (PHH) and non-parenchymal liver cells (NPCs). PHHs (pooled fractions originating from 10 individual donors) and NPCs were purchased from BioIVT (Westbury, NY). Human Liver Microtissues were provided and cultured in InSphero's Akura 96-well plate format. Steatosis was induced either by oleic acid $(400 \mu \mathrm{M}$ coupled to BSA at a molar ratio of 6:1) for 7 days or by medium supplementation of fractionated LDL (Lee Biolsolutions, MO, USA) for 4 days. 3D InSight Human Liver Microtissues, 3D InSight Human Liver Maintenance Medium (TOX) were obtained from InSphero AG, Schlieren, Switzerland.

Culture media. According to the desired phenotype, the three experimental groups of liver PHHs and NPCs co-culture (3D microtissues produced by InSphero) were treated with two different culture media (Fig. 1c). These same media are used to obtain the starting (i.e. Day1) lean and steatotic phenotypes. The LEAN and SOD were kept in a physiological medium (fasting medium), based on William's E, additionally containing glucose, insulin, glucagon, pyruvate and lactate, for 14 days, resulting in a lean phenotype. A second, more anabolic medium (diabetic medium) is used to obtain the steatotic phenotype, based on William's E additionally containing glucose/fructose, insulin as well as plasma fractionated LDL. The SOF is cultured in this medium for 14 days but washed and measured within fasting medium to allow for a direct comparison among experimental groups (see protocol below). Medium exchange was performed simultaneously in each group every 2-3 days as indicated in Fig. $1 \mathrm{~d}$.

Preparation of MTs for confocal imaging. MTs were transferred with a tip quickly coated in fetal calf serum (FCS) into a CellCarrier-384 Ultra Microplates (6057300, Perkin Elmer). They were then fixed in 4\% PFA (P6148, Sigma-Aldrich) for $1 \mathrm{~h}$ at room temperature. After washing with PBS, MTs were stained with Nile Red for lipid droplets, Hoechst for nuclei and CellMask (C10046, ThermoFisher) for plasma membrane for $1 \mathrm{~h}$ at room temperature on an orbital shaker. Subsequently, MTs were washed with and left in PBS until imaging.

Confocal imaging. A Visitron spinning disc confocal microscope was used to acquire the images in Fig. 1ab. In order to acquire the whole tissue, the focus was set to the bottom of the plate and the maximum z-range was imaged. Each acquired channel, its excitation/emission wavelength, and the corresponding exposure time are: (1) confDAPI, 405/461 nm, $250 \mathrm{~ms}$; (2) confGFP, 488/525 nm, $250 \mathrm{~ms}$; (3) confCy5, 640/666 nm, $250 \mathrm{~ms}$.

Protocol for read-out of sample aliquots. Prior read-out, aliquots of 7 MTs are transferred from their original 96 well plate to a single Petri dish where they are immersed in the fasting medium. Such transfer is done by using a standard $200 \mu \mathrm{L}$ pipette. In the case of the SOF, where the 96 well plate contains the MTs in diabetic medium, the aliquots are washed in fasting medium before being placed in the final Petri dish from which they are picked up for measurement (also containing fasting medium). For LEAN and SOD, since they are already immersed in fasting medium during culture, there is no washing step. After the transfer is done under a laminar flow, the culture plate is put back in the incubator and the Petri containing the aliquots is placed on a heated stage set at $37^{\circ} \mathrm{C}$ in laboratory atmosphere. From this point, samples are sequentially transferred from the Petri dish to the CMOS-based NMR sensors, which were previously filled with fasting medium. This second transfer is done by using a $300 \mu \mathrm{m}$ pipette for embryology by Cook Medical and with the help of a stereomicroscope. Once 
a MT is loaded into the sensor and the measurement starts, the successive MT is loaded into a second identical sensor and prepared for the next experiment. By alternating between two sensors it is possible to reduce the experimental time and therefore the exposure of MTs to the laboratory atmosphere. Overall, this procedure took less than $90 \mathrm{~min}$ for all aliquots analyzed. During read-out the three experimental groups LEAN, SOD, SOF are treated separately, sequentially, and in no particular order. Out of the 7 samples picked for the study, some were occasionally lost therefore reducing the experimental points to 6 or 5 in a few time points (see Fig. 4 legend for MTs number).

Received: 6 August 2020; Accepted: 13 October 2020

Published online: 22 December 2020

\section{References}

1. Callaghan, P. T. Principles of Nuclear Magnetic Resonance Microscopy (Oxford University Press on Demand, Oxford, 1993).

2. De Graaf, R. A. In Vivo NMR Spectroscopy: Principles and Techniques (Wiley, New York, 2019).

3. Damadian, R. Tumor detection by nuclear magnetic resonance. Science 171, 1151-1153. https://doi.org/10.1126/scien ce.171.3976.1151 (1971).

4. Le Bihan, D. et al. MR imaging of intravoxel incoherent motions: application to diffusion and perfusion in neurologic disorders. Radiology 161, 401-407. https://doi.org/10.1148/radiology.161.2.3763909 (1986).

5. Hernandez-Baixauli, J. et al. Detection of early disease risk factors associated with metabolic syndrome: a new era with the NMR metabolomics assessment. Nutrients https://doi.org/10.3390/nu12030806 (2020).

6. Kuo, Y. T. et al. In vivo proton magnetic resonance spectroscopy of large focal hepatic lesions and metabolite change of hepatocellular carcinoma before and after transcatheter arterial chemoembolization using 3.0-T MR scanner. J. Magn. Reson. Imaging 19, 598-604. https://doi.org/10.1002/jmri.20046 (2004).

7. Lin, G., Keshari, K. R. \& Park, J. M. Cancer metabolism and tumor heterogeneity: imaging perspectives using MR Imaging and spectroscopy. Contrast Media Mol. Imaging 2017, 6053879. https://doi.org/10.1155/2017/6053879 (2017).

8. Mao, X. A. et al. Fast detection of choline-containing metabolites in liver using $2 \mathrm{D}(1) \mathrm{H}-(1)(4) \mathrm{N}$ three-bond correlation (HN3BC) spectroscopy. J. Magn. Reson. 214, 352-359. https://doi.org/10.1016/j.jmr.2011.11.019 (2012).

9. Knickmeyer, R. C. et al. A structural MRI study of human brain development from birth to 2 years. J. Neurosci. 28, 12176-12182. https://doi.org/10.1523/JNEUROSCI.3479-08.2008 (2008).

10. Plaks, V., Kalchenko, V., Dekel, N. \& Neeman, M. MRI analysis of angiogenesis during mouse embryo implantation. Magn. Reson. Med. 55, 1013-1022. https://doi.org/10.1002/mrm.20881 (2006).

11. Aguayo, J. B., Blackband, S. J., Schoeniger, J., Mattingly, M. A. \& Hintermann, M. Nuclear magnetic resonance imaging of a single cell. Nature 322, 190-191. https://doi.org/10.1038/322190a0 (1986).

12. Grant, S. C. et al. NMR spectroscopy of single neurons. Magn. Reson. Med. 44, 19-22. https://doi.org/10.1002/1522-2594(20000 7) $44: 1 \% 3 c 19:: a i d-m r m 4 \% 3 e 3.0 . c o ; 2-f(2000)$.

13. Fugariu, I. et al. Towards single egg toxicity screening using microcoil NMR. Analyst 142, 4812-4824 (2017).

14. Wong, A. et al. muHigh resolution-magic-angle spinning NMR spectroscopy for metabolic phenotyping of Caenorhabditis elegans. Anal. Chem. 86, 6064-6070. https://doi.org/10.1021/ac501208z (2014).

15. Grisi, M. et al. NMR spectroscopy of single sub-nL ova with inductive ultra-compact single-chip probes. Sci. Rep. 7, 44670. https ://doi.org/10.1038/srep44670 (2017).

16. Messner, S. et al. Transcriptomic, proteomic, and functional long-term characterization of multicellular three-dimensional human liver microtissues. Appl. in Vitro Toxicol. 4, 1-12 (2018).

17. Mukherjee, S. et al. Development and validation of an in vitro 3D model of NASH with severe fibrotic phenotype. Am. J. Transl. Res. 11, 1531-1540 (2019).

18. Hall, K. C. et al. sGC stimulator praliciguat suppresses stellate cell fibrotic transformation and inhibits fibrosis and inflammation in models of NASH. Proc. Natl. Acad. Sci. U. S. A. 116, 11057-11062. https://doi.org/10.1073/pnas.1821045116 (2019).

19. Todhunter, M. E. et al. Programmed synthesis of three-dimensional tissues. Nat. Methods 12, 975-981. https://doi.org/10.1038/ nmeth.3553 (2015).

20. Angulo, P. Nonalcoholic fatty liver disease. N. Engl. J. Med. 346, 1221-1231. https://doi.org/10.1056/NEJMra011775 (2002).

21. Cohen, J. C., Horton, J. D. \& Hobbs, H. H. Human fatty liver disease: old questions and new insights. Science 332, 1519-1523. https://doi.org/10.1126/science.1204265 (2011).

22. Fabbrini, E., Sullivan, S. \& Klein, S. Obesity and nonalcoholic fatty liver disease: biochemical, metabolic, and clinical implications. Hepatology 51, 679-689. https://doi.org/10.1002/hep.23280 (2010).

23. Lavoie, J. M. \& Gauthier, M. S. Regulation of fat metabolism in the liver: link to non-alcoholic hepatic steatosis and impact of physical exercise. Cell Mol. Life Sci. 63, 1393-1409. https://doi.org/10.1007/s00018-006-6600-y (2006).

24. Chiappini, F. et al. Metabolism dysregulation induces a specific lipid signature of nonalcoholic steatohepatitis in patients. Sci. Rep. 7, 46658. https://doi.org/10.1038/srep46658 (2017).

25. Puri, P. et al. A lipidomic analysis of nonalcoholic fatty liver disease. Hepatology 46, 1081-1090. https://doi.org/10.1002/hep.21763 (2007).

26. Brehm-Stecher, B. F. \& Johnson, E. A. Single-cell microbiology: tools, technologies, and applications. Microbiol. Mol. Biol. Rev. 68, 538-559. https://doi.org/10.1128/MMBR.68.3.538-559.2004 (2004).

27. Embade, N. \& Millet, O. Molecular determinants of chronic liver disease as studied by NMR-metabolomics. Curr. Top. Med. Chem. 17, 2752-2766. https://doi.org/10.2174/1568026617666170707124539 (2017).

28. Miccheli, A. et al. Urinary (1)H-NMR-based metabolic profiling of children with NAFLD undergoing VSL\#3 treatment. Int. J. Obes. (Lond.) 39, 1118-1125. https://doi.org/10.1038/ijo.2015.40 (2015).

29. Montinaro, E. et al. 3D printed microchannels for sub-nL NMR spectroscopy. PLoS ONE 13, e0192780. https://doi.org/10.1371/ journal.pone.0192780 (2018).

30. Hockings, P. D. et al. Rapid reversal of hepatic steatosis, and reduction of muscle triglyceride, by rosiglitazone: MRI/S studies in Zucker fatty rats. Diabetes Obes. Metab. 5, 234-243. https://doi.org/10.1046/j.1463-1326.2003.00268.x (2003).

31. Bollard, M. E. et al. High-resolution (1) $\mathrm{H}$ and (1)H-(13)C magic angle spinning NMR spectroscopy of rat liver. Magn. Reson. Med. 44, 201-207. https://doi.org/10.1002/1522-2594(200008)44:2\%3c201::aid-mrm6\%3e3.0.co;2-5 (2000).

32. Fernando, H. et al. (1)H and (3)(1)P NMR lipidome of ethanol-induced fatty liver. Alcohol Clin. Exp. Res. 34, 1937-1947. https:// doi.org/10.1111/j.1530-0277.2010.01283.x (2010).

33. Guillén, M. D. \& Ruiz, A. Rapid simultaneous determination by proton NMR of unsaturation and composition of acyl groups in vegetable oils. Eur. J. Lipid Sci. Technol. 105, 688-696 (2003). 
34. Caussy, C., Reeder, S. B., Sirlin, C. B. \& Loomba, R. Noninvasive, quantitative assessment of liver fat by MRI-PDFF as an endpoint in NASH trials. Hepatology 68, 763-772. https://doi.org/10.1002/hep.29797 (2018).

35. Andersen, T., Gluud, C., Franzmann, M. B. \& Christoffersen, P. Hepatic effects of dietary weight loss in morbidly obese subjects. J. Hepatol. 12, 224-229. https://doi.org/10.1016/0168-8278(91)90942-5 (1991).

36. Grisi, M., Gualco, G. \& Boero, G. A broadband single-chip transceiver for multi-nuclear NMR probes. Rev. Sci. Instrum. 86, 044703. https://doi.org/10.1063/1.4916206 (2015).

\section{Acknowledgements}

We thank Margaux Duchamp from LMIS4 laboratory (EPFL) for allowing use of cell culture incubators. We thank prof. Lyndon Emsley for fruitful discussions. We are grateful to the help of the staff at the Center of Micro and Nano Technology (CMi) at EPFL with the fabrication process. This work was partially supported by EPFL, the Swiss National Science Foundation under grant 40B1-0_180268, the FIT foundation of Canton Vaud via the EPFL Innogrant program, Innosuisse under Grant 39821.1 IP-LS, and the European Union's Horizon 2020 research and innovation program under Grant agreement No 681002.

\section{Author contributions}

G.M.C., M.G., J.L., W.M. conceived the study. L.E., W.M., J.L. provided samples and protocols. M.G. and G.M.C. designed and fabricated the CMOS-based sensors. M.G. and G.M.C. performed experiments and data collection. G.B., J.B. provided critical analysis and discussion. M.G., K.J.R., E.R. performed data analysis. M.G. supervised the project. M.G. and K.J.R. wrote the manuscript. All authors edited and approved the content.

\section{Competing interests}

MG, GMC, JB, and GB are the authors of a patent application related to this work (Submitted on September 24th 2018, PCT/IB2018/057348). GMC and MG are co-founders of Annaida Technologies, which owns an exclusive license of the mentioned patent application. The authors declare no competing interests.

\section{Additional information}

Supplementary information is available for this paper at https://doi.org/10.1038/s41598-020-75480-0.

Correspondence and requests for materials should be addressed to M.G.

Reprints and permissions information is available at www.nature.com/reprints.

Publisher's note Springer Nature remains neutral with regard to jurisdictional claims in published maps and institutional affiliations.

Open Access This article is licensed under a Creative Commons Attribution 4.0 International License, which permits use, sharing, adaptation, distribution and reproduction in any medium or format, as long as you give appropriate credit to the original author(s) and the source, provide a link to the Creative Commons licence, and indicate if changes were made. The images or other third party material in this article are included in the article's Creative Commons licence, unless indicated otherwise in a credit line to the material. If material is not included in the article's Creative Commons licence and your intended use is not permitted by statutory regulation or exceeds the permitted use, you will need to obtain permission directly from the copyright holder. To view a copy of this licence, visit http://creativecommons.org/licenses/by/4.0/.

(C) The Author(s) 2020 